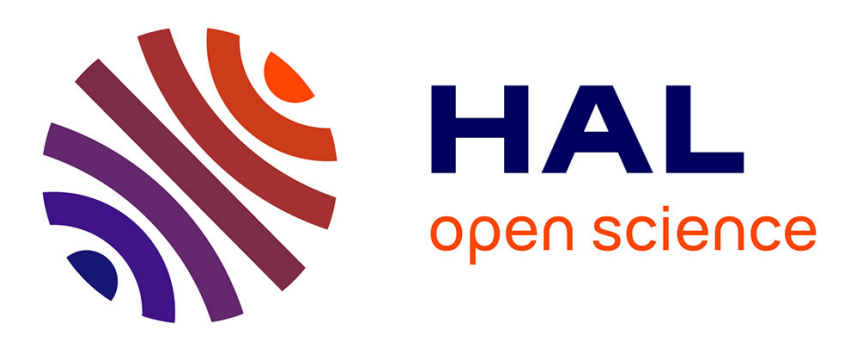

\title{
Observation of unbroadened giant quantum oscillations in the attenuation of rayleigh waves in gallium. surface stress effect
}

N. Duruy, G. Bellessa

\section{- To cite this version:}

N. Duruy, G. Bellessa. Observation of unbroadened giant quantum oscillations in the attenuation of rayleigh waves in gallium. surface stress effect. Journal de Physique Lettres, 1976, 37 (6), pp.153-156. 10.1051/jphyslet:01976003706015300 . jpa-00231262

HAL Id: jpa-00231262

https://hal.science/jpa-00231262

Submitted on 1 Jan 1976

HAL is a multi-disciplinary open access archive for the deposit and dissemination of scientific research documents, whether they are published or not. The documents may come from teaching and research institutions in France or abroad, or from public or private research centers.
L'archive ouverte pluridisciplinaire HAL, est destinée au dépôt et à la diffusion de documents scientifiques de niveau recherche, publiés ou non, émanant des établissements d'enseignement et de recherche français ou étrangers, des laboratoires publics ou privés. 
Classification

Physics Abstracts

$7.270-7.830-8.216$

\title{
OBSERVATION OF UNBROADENED GIANT QUANTUM OSCILLATIONS IN THE ATTENUATION OF RAYLEIGH WAVES IN GALLIUM. SURFACE STRESS EFFECT
}

\author{
N. DURUY and G. BELLESSA \\ Laboratoire de Physique des Solides (*), \\ Université Paris-Sud, 91405 Orsay, France \\ (Reçu le 20 février 1976, révisé le 13 avril 1976, accepté le 15 avril 1976)
}

\begin{abstract}
Résumé. - Les oscillations quantiques géantes de l'atténuation des ondes de Rayleigh sont étudiées sur une surface métallique non contrainte et contrainte. Dans le premier cas le pic d'atténuation se comporte suivant les prévisions théoriques. Un tel pic non élargi est observé pour la première fois. Si la surface est contrainte, le pic d'atténuation subit un élargissement inhomogène qui est expliqué en tenant compte du champ de déformation au voisinage de la surface.
\end{abstract}

\begin{abstract}
Giant quantum oscillations in the attenuation of Rayleigh waves are studied on an unstressed metal surface and on a stressed one. In the first case the attenuation peak behaves according to the theory. Such an unbroadened peak is reported for the first time. If the metal surface is stressed, the attenuation peak suffers an inhomogeneous broadening which is explained by taking into account the deformation field near the surface.
\end{abstract}

Giant quantum oscillations (GQO) in the attenuation of Rayleigh waves have been already observed [1]. However the linewidth and its variation as a function of the temperature were not in agreement with the theory of Grishin and Kaner [2]. In this paper we report the observation of unbroadened attenuation peaks which agree with the theory [2]. To obtain such unbroadened peaks, the metal surface must be free of any stress. As the surface is deliberately stressed the attenuation peaks are largely broadened. Experimental evidence for this effect is reported and a qualitative explanation is proposed.

The experimental procedure has been previously described [3]. The single crystal is made with very pure gallium in a plastic mold which has two polished faces [4]. The flat surfaces of the gallium crystal are normal to the $\mathbf{b}$ axis [5] and to the magnetic field. Two rectangular $\mathrm{ZnS}$ films about $6000 \AA$ thick are evaporated on one crystal face. These piezoelectric films are at a distance of $5 \mathrm{~mm}$ from each other and support two evaporated metallic combs. The comb teeth are $20 \mu \mathrm{m}$ wide and at a distance of $40 \mu \mathrm{m}$ from each other. Rayleigh waves are generated with one comb and detected with the second one, using a pulse technique. This classical technique has been modified in order to allow the use of a phase sensitive system and to enhance in this way the signal to noise ratio.

(*) Laboratoire associé au C.N.R.S.
However, there is an important difficulty about the phase detection of the delayed pulse on the second comb. This difficulty arises because the Rayleigh wave velocity varies in a similar way as the attenuation, when the magnetic field is swept. The relative velocity change is small (about $5 \times 10^{-4}$ in the present experiments), but it is sufficient to cause a relative phase change of the delayed pulse of about $20^{\circ}$ to $40 \mathrm{MHz}$. To resolve the problem, an auxiliary phase detector is used and its output drives the RF-generator frequency so that the phase difference between the reference signal and the delayed pulse is kept constant. A more detailed description of this technique will be published elsewhere. In order to display the effect of stresses on the GQO of Rayleigh waves, the metal surface between the two combs is firstly studied free of any stress (except the stresses which may arise on account of the presence of a thin oxide film). Then, a $\mathrm{ZnS}$ film of variable thickness is evaporated on the surface between the two combs.

The GQO in the attenuation of Rayleigh waves appear at magnetic fields above $20 \mathrm{kOe}$. They have a spikelike character. The peaks are periodic in inverse magnetic field. The measured period is $29.7 \times 10^{-7} \mathrm{Oe}^{-1}$ and is equal to the period of the GQO in the attenuation of acoustic bulk waves in longitudinal magnetic field [6]. The Rayleigh wave velocity exhibits also GQO. These oscillations are similar to those which have been observed in the bulk [7]. Figure 1 shows the shape of the attenuation 


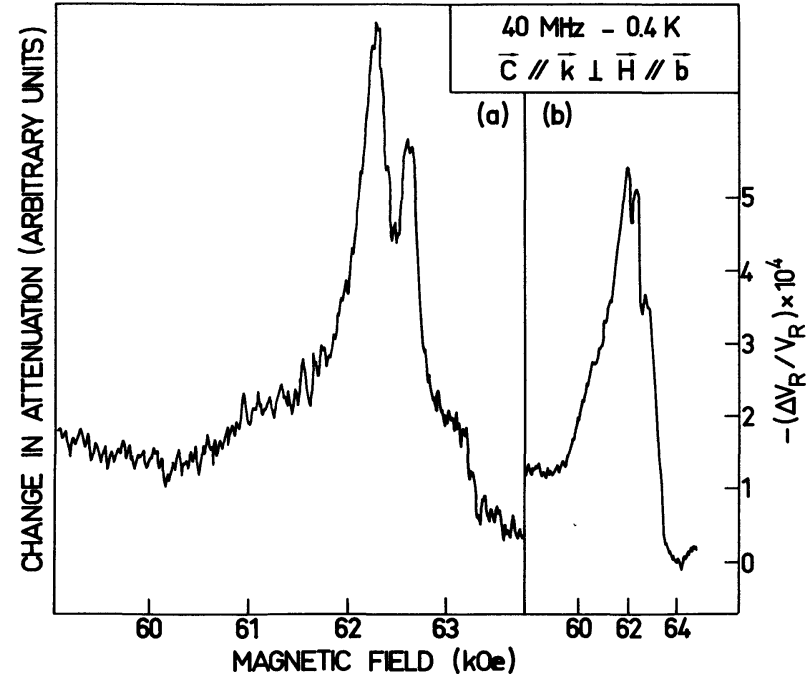

FIG. 1. - Recorder tracing of the change in the attenuation and the velocity of Rayleigh waves as a function of magnetic field. The metal surface is free of any stress.

peak and the velocity peak for a sample with a free surface. It appears clearly on figure $1 a$ that a small broadened line is superposed on the main peak. The former line arises from the GQO on the stressed surface under the combs since there are always $\mathrm{ZnS}$ films on these parts of the sample. This assertion will become evident later on. In the present experiments, the propagation length under the combs is $30 \%$ of the total propagation length. The main peak of figure $1 a$ exhibits a spin splitting as in the bulk case [6]. The splitting of the peak (330 Oe) and the linewidth at half-height $(600 \mathrm{Oe})$ have the same values as those obtained with acoustic bulk waves at the same temperature [1]. However the line shape presents some peculiarities. Firstly the amplitudes of the two subpeaks are appreciably different. Secondly the line shape is asymmetrical, the high-field side being steeper than the low-field side. The linewidth reported here is to be compared with the $1500 \mathrm{Oe}$ linewidth in reference [1].

The attenuation peak is broadened if the sample surface is stressed. The resulting curves are reported on figure 2. Curve $2 a$ is obtained from a sample which has been previously subjected to chemical treatment. It is necessary to make such treatment, otherwise the film-sample adhesion is not good and a peeling of the evaporated films appears after a thermal cycle. The chemical treatment consists in diping the gallium crystal in hydrofluoric acid for $2 \mathrm{~min}$. Consequently a thin fluoride film is created on the sample. It is difficult to estimate the thickness of this film but it is certainly less than $500 \AA$. When the temperature is lowered down to $0.4 \mathrm{~K}$, stresses on the metal surface arise from the difference in the thermal-expansion coefficients of the film and the gallium crystal. Curve $2 a$ exhibits a slight broadening in comparison with the peak on figure $1 a$. The spin splitting is still visible through the trapezoidal shape of the top of the peak. Taking advantage of this particular shape, the linewidth can be calculated with the parameters of the undeformed curve (Fig. 1a). It is found to be $700 \mathrm{Oe}$. This value is consistent with the linewidth $(750 \mathrm{Oe})$ which is measured on curve $2 a$ after the small broadened line of figure $1 a$ has been substracted from the experimental peak (this line still appears on figure 2 through a slope change on the low field side of the peak). Curve $b$ on figure 2 is obtained after a $6000 \AA$ thick film of $\mathrm{ZnS}$ has been evaporated on the sample surface. In this case, there is no longer the problem of inhomogeneous stresses on the surface since the thickness of the $\mathrm{ZnS}$ film is the same under the combs and between them. It appears on figure 2 that the attenuation peak is widely broadened. Its width at half-height is $1500 \mathrm{Oe}$ and is to be compared with $600 \mathrm{Oe}$ which is the linewidth of the unstressed surface at the same temperature (Fig. 1a). The temperature dependence of the GQO of Rayleigh waves has been also studied with a sample surface unstressed and stressed respectively. In the case of the unstressed surface the attenuation peak of Rayleigh waves behaves like the attenuation peak of acoustic bulk waves. That is to say, the

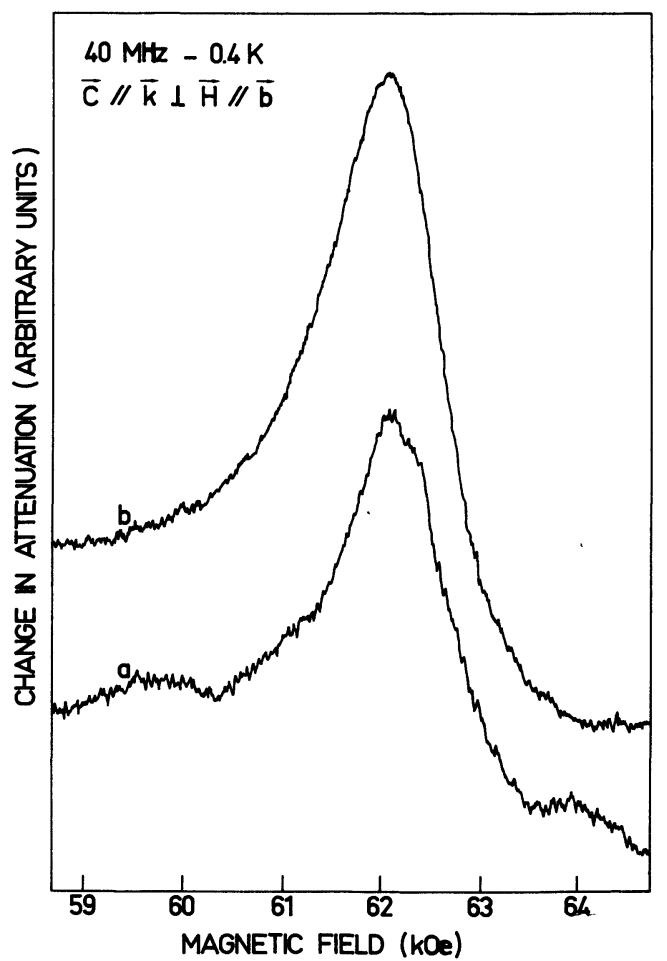

FIG. 2. - Recorder tracing of a GQO peak showing the line broadening as the surface is stressed. Curve $a$ is for a surface which has been subjected to chemical treatment (see text). Curve $b$ is obtained after a $6000 \AA$ thick film of $\mathrm{ZnS}$ has been evaporated on the sample surface.

splitting of the peak disappears when the temperature is raised to $1.2 \mathrm{~K}$, then the linewidth increases when the temperature is further raised to $4.17 \mathrm{~K}$ [1]. In the case of the stressed surface (with a $6000 \AA$ thick $\mathrm{ZnS}$ film) the line shape is not very sensitive to the temperature variation. It is independent of the temperature 
between 0.4 and $1.2 \mathrm{~K}$. Then the linewidth slightly increases and reaches 1800 Oe at $4.17 \mathrm{~K}$.

The experimental results in the case of the unstressed surface can be explained by the theory of Grishin and Kaner [2]. These authors have studied the shape of the absorption line and have considered temperature effects. The latter induces a line broadening which is similar to the thermal broadening in the case of bulk waves. If the temperature is the single cause of broadening, the peak has a symmetrical shape and its width at half-height can be written as in the bulk case [6]

$$
\delta H=3.53 k T H P m_{\mathrm{c}} c / e \hbar
$$

where $P$ is the period in $1 / H$ and $m_{\mathrm{c}}$ is the cyclotron mass $\left(m_{\mathrm{c}}=0.06 m_{0}\right.$ in the present case). The experimental value (Fig. 1a) is in agreement with eq. (1) (taking the spin splitting into account). This agreement is noteworthy since, experimentally the reflections of the electrons on the surface are mostly diffuse and the theory has been made in the case of specular reflections. The experimental results in the case of the stressed surface (Fig. 2) cannot be explained with the theory of Grishin and Kaner. However, it is possible to explain the line broadening by taking into account the strains near the surface. In order to estimate the deformation at the film-gallium interface, the thermal expansion coefficients $\alpha$ are used. $\alpha$ is known down to $20 \mathrm{~K}$ for $\mathrm{ZnS}$ [8] and down to $4.17 \mathrm{~K}$ for $\mathrm{Ga}$ [9]. If the $\mathrm{ZnS}$ film is supposed stronger than the gallium single crystal, it imposes the strain in the gallium. In this case the linear strain of the gallium surface is

$$
\varepsilon=\int_{300}^{1}\left(\alpha_{\mathrm{Ga}}-\alpha_{\mathrm{ZnS}}\right) \mathrm{d} T \cong-3 \times 10^{-3}
$$

where $300 \mathrm{~K}$ is the temperature of the substrate during the deposition of the $\mathrm{ZnS}$ film. It is interesting to compare this value with the coefficient of linear compressibility for $\mathrm{Ga}$ [10]

$$
\chi \cong 0.5 \times 10^{-3} \mathrm{kbar}^{-1} \text {. }
$$

Eq. (2) shows that the crystal is stretched at the interface. However it gives only an order of magnitude for the deformation, since it neglects any strain in the $\mathrm{ZnS}$ film. Obviously, the dilatation $\Delta(z)$ at a distance $z$ from the interface decreases as $z$ increases and the lattice is inhomogeneously deformed. The Rayleigh waves are confined to a layer approximatively one wavelength thick and so they interact with the electrons which move in the strained part of the crystal. Hence, any change in the behaviour of the conduction electrons, appears on the GQO of Rayleigh waves. Hunter and Nabarro have considered the propagation of electrons in an inhomogeneously deformed lattice [11]. They obtain for the electron energy an expression of the form

$$
E(k, z)=E \Delta(z)+\hbar^{2} k^{2} / 2 m
$$

where $\Delta$ is the dilation which depends on the spatial coordinates. In the present case it is taken as $z$-dependent. The effect of the magnetic field is to quantize the last term of eq. (3). Then, the electron energy is

$$
E_{n}\left(k_{z}, z\right)=E \Delta(z)+n \hbar \Omega+\hbar^{2} k_{z}^{2} / 2 m_{z}
$$

where the magnetic field is in the $z$-direction, $n$ is the Landau level number, $\Omega$ is the cyclotron frequency, $k_{z}$ is the $z$-component of the electron momentum along the magnetic field and $m_{z}$ is the effective mass in the magnetic field direction. Since the chemical potential is the same throughout the metal, it follows from eq. (4) that the magnetic field value for which the energy of a given Landau level equals the chemical potential depends on the spatial coordinates. Hence, it is no longer a single Landau level which gives rise to an attenuation peak but rather a Landau level distribution. Thus, the attenuation peak suffers an inhomogeneous broadening. If the width of the Landau level distribution is much larger than $k T$, the linewidth is no more dependent on the temperature. The experimental results for the stressed surface (Fig. 2) can be explained with the preceding effect. A quantitative theory of this broadening effect will be published elsewhere.

The present experiments have shown that it is possible to obtain unbroadened GQO in the attenuation of Rayleigh waves. In order to obtain such lines, the sample surface must be free of any stress. Then, the experimental linewidth agrees with the theory [2]. This agreement is noteworthy since the theory has been carried out in the case of specular reflections of the electrons on the surface. However, the asymmetrical line shape reported here cannot be explained with the theory [2]. This peculiar shape is perhaps due to diffuse reflections of the electrons on the surface (as the asymmetrical line shape in the case of GQO in the attenuation of acoustic bulk waves is due to collisions of the electrons with the impurities) [12]. If the surface is stressed, the GQO suffer an inhomogeneous broadening which has been explained by taking into account the deformation field near the surface. Similar considerations have been used to explain the broadening of the GQO in the attenuation of acoustic bulk waves in a dislocated lattice [13]. In the present paper we have not considered the dislocations near the surface. However, they can act also on the line broadening. Thin films exhibit indeed high tensile strength, due to the high density of defects frozen during the deposition and a plastic flow may occur in the substrate near the interface as the sample is cooled. Unfortunately the tensile strength is not known for $\mathrm{Ga}$ at low temperature and it is not possible presently to estimate the magnitude of this effect. Further experiments are in progress to probe precisely the deformation field near the surface by considering the Rayleigh frequency and the thermal-expansion coefficient of the evaporated film. 


\section{References}

[1] Bellessa, G., Phys. Rev. Lett. 34 (1975) 1392.

[2] Grishin, A. M. and Kaner, E. A., Zh. Eksp. \& Teor. Fiz. 65 (1973) 735; Sov. Phys. JETP 38 (1974) 365.

[3] Bellessa, G., J. Physique Lett. 36 (1975) L-137.

[4] The authors are indebted to P. de la Bretèque of Alusuisse France S. A. for the kind supplying of very pure gallium $(99.9999 \%$ at least).

[5] The $\mathbf{a}, \mathbf{b}$, and $\mathbf{c}$ axes are the crystallographic axes of the gallium crystal with the usual notations.

[6] Shapira, Y. and Lax, B., Phys. Rev. A 138 (1965) 1191.

[7] Neuringer, L. J. and Shapira, Y., Phys. Rev. 165 (1968) 751.
[8] Melins, G., Handbuch der anorganischen Chemie (1956).

[9] Yaqub, M. and Cochran, J. F., Phys. Rev. A 137 (1965) 1182.

[10] Galkin, A. A., Popovich, A. I., Degtyar, E. P. and D'yaCHENKo, A. I., Zh. Eksp. \& Teor. Fiz. 68 (1975) 711 ; Sov. Phys. JETP 41 (1975) 352.

[11] Hunter, S. C. and Nabarro, F. R. N., Proc. R. Soc. Lond. A 220 (1953) 542.

[12] Our samples are made with very pure Gallium and there is no impurity effect on the line shape of the GQO of acoustic bulk waves down to $0.4 \mathrm{~K}$ as shown in reference [1].

[13] Bellessa, G., Phys. Rev. B 7 (1973) 2400. 